\title{
Hypothermia as an Adjunctive Treatment in Pediatric Patients with Naegleria fowleri: A Systematic Review
} \author{
Eric S Nussbaum, $M D^{2 *}$ \\ ${ }^{1}$ Medibio Limited, United States HQ, 8696 USA \\ ${ }^{2}$ Department of Neurosurgery, National Brain Aneurysm \& Tumor Center, United Hospital, USA \\ ${ }^{3}$ Superior Medical Experts, USA
}

Archie Defillo, $M D^{1,2 \#}$, Penelope E Nussbaum², Parvathy Hariharan, $M S^{3}$, Alexander Mebane, $B S^{3}$ and

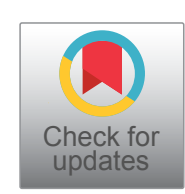

\begin{abstract}
We reviewed the literature for pediatric patients infected with Naegleria fowleri ( $N$. fowleri) to assess the relationship between patient survival and treatment with hypothermia. A systematic literature search was performed in PubMed to identify studies describing cases of pediatric $N$. fowleri infections. We identified 47 studies describing 55 pediatric patients (median age 9 -years [range: 5 months-16-years]; $22.6 \%$ female [12/53]) with a mortality rate of $85.5 \%(47 / 55)$. Among these studies, 3 patients (33.3\% female; median age 12-years [range: 12-14]) were treated with induced hypothermia in conjunction with other pharmaceutical and surgical interventions. Two of these patients succumbed to the illness, while the other made a full neurologic recovery. Our results indicate that therapeutic hypothermia has not been used widely in pediatric patients infected with $N$. fowleri. Further studies are needed to determine whether hypothermia reduces pathogenicity; increased physician awareness of this illness is also needed.
\end{abstract}

\section{Keywords}

Naegleria fowleri, Amoeba, Central nervous system protozoal infections, Primary amebic meningoencephalitis

\section{Abbreviations}

N. fowleri: Naegleria fowleri; PAM: Primary Amebic Meningoencephalitis; CSF: Cerebrospinal Fluid; CT: Computerized Tomography; MRI: Magnetic Resonance Imaging

\section{Introduction}

Naegleria fowleri ( $N$. fowleri) is a pathogenic amoeba that causes primary amebic meningoencephalitis (PAM), a very rare but nearly always fatal central nervous system infection $[1,2]$. Up to eight $N$. fowleri cases are diagnosed in the USA per year [1], which often occurs after seasonal water activities such as swimming and water skiing when contaminated water enters the nasal cavity [3]. The pathogenic amoeba attaches to the nasal mucosa, then moves along the olfactory nerve through the cribriform plate [4] and onto the olfactory bulbs, eliciting an intense innate immune response in the central nervous system $[5,6]$. The cribriform plate has increased porosity in children and young adults compared to older populations [4], potentially placing this age group at greater risk for $N$. fowleri infections.

Despite standard treatment regimens, which include antibiotics (i.e., biologically produced substances) and antimicrobials (i.e., synthetically produced substances), PAM has an associated overall mortality rate of over $97 \%$ in adults and children $[1,2]$. A few clinicians have reported using hypothermia as an adjunctive treatment with at least one report showcasing successful recovery and positive neurological outcomes, possibly due to reduction or prevention of cerebral edema [7-9]. We performed a systematic literature review to identify clinical outcomes among patients with $N$. fowleri treated with induced hypothermia.

\section{Materials and Methods}

We conducted a systematic literature review using the

*Corresponding author: Eric S Nussbaum, MD, Department of Neurosurgery, National Brain Aneurysm \& Tumor Center, 3033 Excelsior Boulevard, Suite 495, Minneapolis, MN 55416, USA, Tel: 612-374-0177, Fax: 612-374-0893

Accepted: May 05, 2021

Published online: May 07, 2021

Citation: Defillo A, Nussbaum PE, Hariharan P, et al. (2021) Hypothermia as an Adjunctive Treatment in Pediatric Patients with Naegleria fowleri: A Systematic Review. J Pediatr Neurol Neurosci 5(1):105-110 
Citation: Defillo A, Nussbaum PE, Hariharan P, et al. (2021) Hypothermia as an Adjunctive Treatment in Pediatric Patients with Naegleria fowleri: A Systematic Review. J Pediatr Neurol Neurosci 5(1):105-110

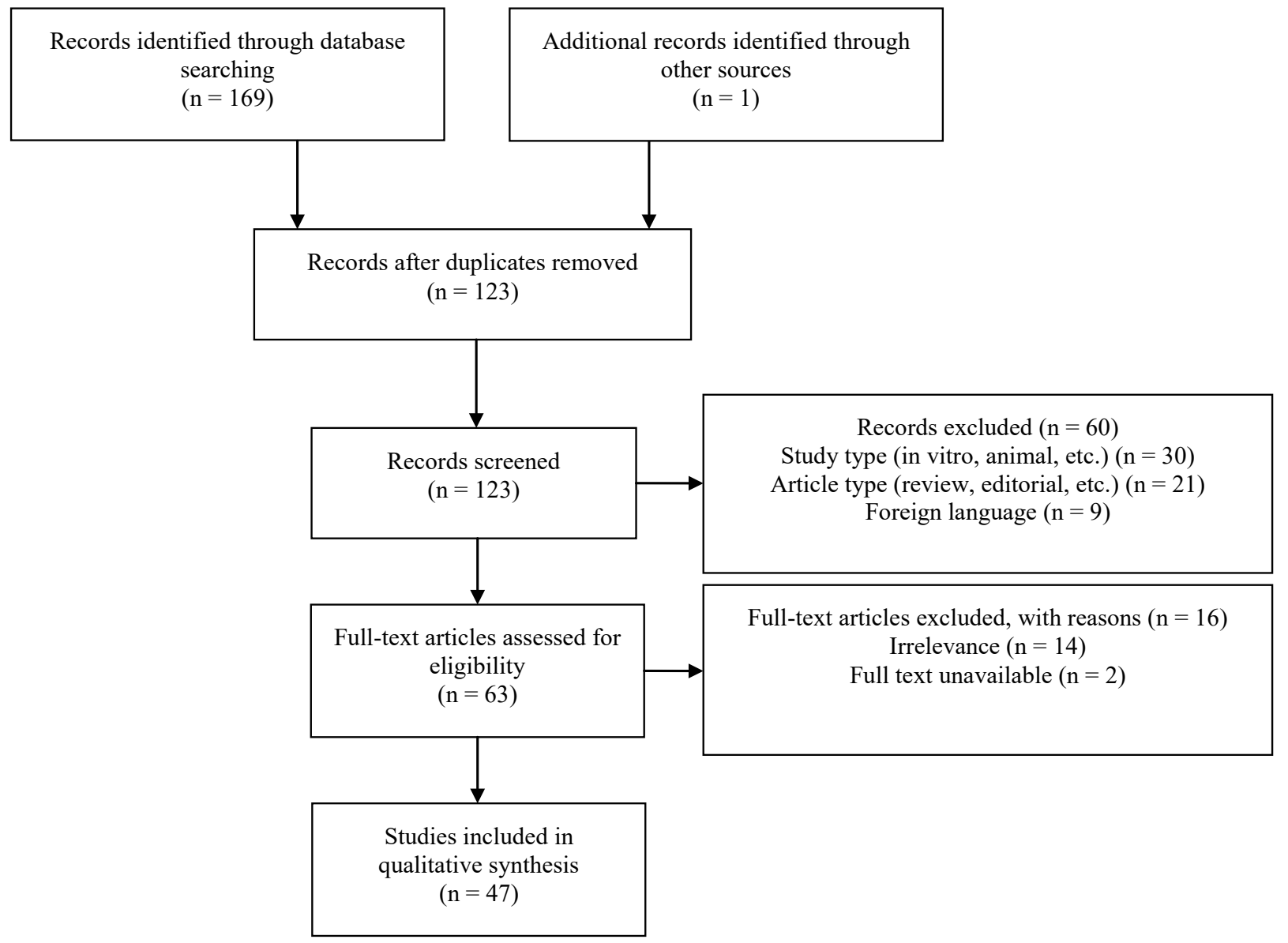

Figure 1: PRISMA flow diagram for systematic literature search.

PubMed database with the following search terms: 1) "Naegleria fowleri" AND (pediatric OR child OR infant), and 2) "Primary amoebic meningoencephalitis" AND (pediatric OR child OR infant). Studies describing pediatric patients with $N$. fowleri and treatment outcomes from 1981-2019 were included, while articles with adult patients above 18 -years, preclinical studies, opinion articles, reviews, and foreign language articles were excluded. Additionally, duplicate articles and those that could not be located in their entirety were excluded. Included articles were examined for consistency with PAM diagnosis (rapidly progressive meningoencephalitis with purulent cerebrospinal fluid [CSF] following water exposure), taking note of any likely inappropriate diagnosis. Included articles were examined for redundant cases. References were scoured for additional relevant articles. Systematic review methods adhered to PRISMA guidelines (Figure 1). The number of patients in each case report or article was documented, along with patient age, gender, treatment regimen(s), and outcome.

\section{Results}

\section{Literature search results}

Excluding duplicates, our literature search yielded 122 articles published between 1981 and 2019, with one article identified from other sources. Of these, 76 were excluded and 47 containing relevant patient-specific data met inclu- sion criteria (Figure 1). The studies were further divided into two groups: 1) Those that treated patients with therapeutic hypothermia + standard therapy ( 5 studies of 3 patients); and 2) Those that used standard therapy alone (42 studies of 52 patients).

\section{Study population: Treatment characteristics and patient outcomes}

The 47 included studies described a total of 55 pediatric patients with $N$. fowleri ( $22.6 \%$ female, $12 / 53$ [as two studies did not identify sex]; median age: 9-years [range: 5 months 16-years]) treated for $N$. fowleri infection, including standard therapies and hypothermia with an overall mortality rate of $85.5 \%(47 / 55)$.

Therapeutic hypothermia: Among these patients, 3 (33.3\% female; median age 12; age range 12-14-years) were treated with therapeutic hypothermia in a hyperbaric chamber, in addition to treatment with pharmaceutical agents or other procedures (Table 1) [7-11]. One study reported patient core body temperature in the hyperbaric chamber, which ranged from $32-34{ }^{\circ} \mathrm{C}$ [7]. The duration and hypothermic treatment was not documented by any study of the specific timing only documented in one study [7-11]. Hypothermia was always combined with medical management and cranial pressure alleviating procedures, such as craniotomy, hyperosmolar therapy, and shunt placement. All 3 patients (100\%, 
Citation: Defillo A, Nussbaum PE, Hariharan P, et al. (2021) Hypothermia as an Adjunctive Treatment in Pediatric Patients with Naegleria fowleri: A Systematic Review. J Pediatr Neurol Neurosci 5(1):105-110

Table 1: Literature review of pediatric $N$. fowleri cases treated with hypothermia.

\begin{tabular}{|c|c|c|c|c|}
\hline Author & $\begin{array}{l}\text { Patient Age } \\
\text { (yrs)/Sex }\end{array}$ & Pharmaceutical Management & $\begin{array}{l}\text { Administered } \\
\text { Treatment }\end{array}$ & Outcome \\
\hline Cope, et al., 2016 [10] & $12 / \mathrm{M}$ & $\begin{array}{l}\text { amphotericin B, azithromycin, } \\
\text { dexamethasone, fluconazole, } \\
\text { miltefosine, rifampin }\end{array}$ & $\begin{array}{l}\text { Surgical decompression, } \\
\text { hypertonic saline and mannitol, } \\
\text { induced hypothermia (no } \\
\text { description) }\end{array}$ & Brain death \\
\hline $\begin{array}{l}\text { Linam, et al., } 2015 \text { [7]; } \\
\text { Dunn, et al., } 2016 \text { [8]; } \\
\text { Heggie, et al., } 2017 \text { [9] }\end{array}$ & $12 / F$ & $\begin{array}{l}\text { amphotericin B, azithromycin, } \\
\text { dexamethasone, fluconazole, } \\
\text { hyperosmolar therapy, } \\
\text { miltefosine, rifampin }\end{array}$ & $\begin{array}{l}\text { External ventricular drain, } \\
\text { CSF drainage, moderate } \\
\text { hyperventilation, induced } \\
\text { hypothermia }\left(32-34^{\circ} \mathrm{C}\right) \text { in the } \\
\text { management of PAM }\end{array}$ & $\begin{array}{l}\text { Full neurologic recovery; } \\
\text { cerebral edema resolved } \\
\text { after using hypothermia } \\
\text { in conjunction with other } \\
\text { therapies }\end{array}$ \\
\hline Stowe, et al., 2017 [11] & $14 / \mathrm{M}$ & $\begin{array}{l}\text { amphotericin B, azithromycin, } \\
\text { ceftriaxone, fluconazole, } \\
\text { miltefosine, pentobarbital, } \\
\text { rifampin, vancomycin }\end{array}$ & $\begin{array}{l}\text { Induced hypothermia (no } \\
\text { description) }\end{array}$ & Death \\
\hline
\end{tabular}

PAM: Primary amebic meningoencephalitis; CSF: Cerebral spinal fluid

$3 / 3)$ received both antibiotics and antimicrobials. Additionally, $100.0 \%(3 / 3)$ received miltefosine, $100.0 \%(3 / 3)$ received amphotericin B, and $100.0 \%(3 / 3)$ received azithromycin. One patient received therapeutic hypothermia, hypertonic saline, mannitol, and surgical decompression at an unspecified time point to manage cerebral edema, but none of these measures resulted in clinical improvement and the patient was declared brain-dead on day 16 in the hospital [10]. Another patient with headache, weakness, and fever was initially sent home from a local hospital after a reportedly normal computed tomography scan, then returned the following day due to onset of vomiting and confusion. This patient received therapeutic hypothermia and pentobarbital in an attempt to aggressively manage intracranial pressure, but these efforts were unsuccessful and the patient expired on day 4 of hospitalization [11]. In the surviving patient, local laboratory protocols allowed for the $N$. fowleri diagnosis to be communicated to medical staff within one hour of receiving the CSF specimen [8]. The patient's CSF specimen was negative for $N$. fowleri by day 3 , and after 52 days of hospitalization, the patient made a full neurological recovery [7].

Standard therapy: Fifty-two patients with $N$. fowleri infections were treated with standard therapies without hypothermia (Supplemental Table 1). Similar to the therapeutic hypothermia group, patients in the standard therapy group were treated variously with ventriculostomy, shunt placement, and intracranial pressure monitors to decrease cerebral edema and alleviate intracranial pressure [12-16]. Among these patients, $7.7 \%(4 / 52)$ received miltefosine, 51.9\% (27/52) received amphotericin $B$, and $17.3 \%(9 / 52)$ received azithromycin. This patient population had a mortality rate of $86.5 \%$ (45/52), including one clinically deteriorating infant who was removed from the hospital against medical advice [17].

Surviving patients in this group were predominately male $(85.7 \%, 6 / 7)$ and had median age 8 years (range: 5 months - 10-years). Six of the seven (85.7\%) survivors made full neurological recoveries $[14,18-22]$. One previously healthy patient continued to be nonverbal with static encephalopathy and seizures 18 months after discharge, despite prolonged support in a 39-day hospitalization and subsequent pediatric rehabilitation for 36 days [10]. All seven (100.0\%) surviving patients received antibiotics and antimicrobials, while only 1/7 (14.3\%) received miltefosine (Supplemental Table 1).

\section{Discussion}

Our literature review and analysis of pediatric patients with $N$. fowleri suggest that hypothermia as an adjunctive therapy for PAM is not exceedingly common and, based on very limited data, has resulted in similar survival and neurological outcomes in comparison with medical management alone. As there is a paucity of data on efficacious treatments for PAM, hypothermia may be worth exploring as an adjunctive therapy when treating pediatric populations for this highly lethal condition.

Unfortunately, the prognosis of $N$. fowleri infection has not appeared to improve over the years, despite pharmaceutical and technological advancements. Of the 17 case reports published pre-2000 in this review, only two survived, resulting in a survival rate of $11.8 \%$. Post- 2000 case reports had a very similar survival rate at $14.3 \%(5 / 35)$. The rarity of PAM, its tendency for delayed diagnosis, and swiftly deteriorating patients effectively preclude the possibility of clinical trials to determine more efficacious treatments; therefore, physicians must base potential treatment plans on case reports, in vitro studies, and sound clinical reasoning. Therapeutic hypothermia is a product of such reasoning and a recent explorative adjunctive therapy, with the earliest case report using this treatment published in 2015 [7].

It is possible that therapeutic hypothermia may exert neuroprotective effects on pediatric patients with $N$. fowleri infections, which result in host cell, nerve, and tissue destruction via the release of cytolytic molecules $[3,5,23]$. The collective effect of pathogenicity and the body's associated immune response results in significant central nervous system damage and often death [3]; notably, increased intracranial pressure and CSF pressure are directly associated with death in N. fowleri infected patients [23]. Elevated intracranial pressure is commonly managed via intracranial pressure 
Citation: Defillo A, Nussbaum PE, Hariharan P, et al. (2021) Hypothermia as an Adjunctive Treatment in Pediatric Patients with Naegleria fowleri: A Systematic Review. J Pediatr Neurol Neurosci 5(1):105-110

monitor placement $[15,16]$, ventriculostomy $[12,24-26]$, craniectomy [27], shunt [14], and other surgical decompression techniques [10]; hypothermia may offer additional neuroprotective benefits against brain damage due to intracranial pressure through its involvement with inflammation, metabolism, free-radical production, apoptosis, neurogenesis, and angiogenesis [28]. Hypothermia may also exert protective effects by arresting the development and/or pathogenicity of microbes. In vitro studies have shown that $N$. fowleri enters an active growth phase at $42{ }^{\circ} \mathrm{C}$, and cell density is reduced $32{ }^{\circ} \mathrm{C}[29,30]$. Likewise, when temperatures rise above $35^{\circ} \mathrm{C}$, the stable cyst form of $N$. fowleri becomes more invasive [31]. Thus, it is possible that lower body temperatures induced by therapeutic hypothermia may inhibit active microbial growth. In the setting of nearly universal fatality, it is certainly reasonable to explore hypothermia as a useful adjunctive treatment.

Nevertheless, one of the biggest obstacles standing in the way of implementing new or potentially effective therapies for $N$. fowleri infection is delayed diagnosis. The Centers for Disease Control and Prevention estimate that over three-quarters of infected patients are diagnosed after death [2]. Diagnosis is primarily by analysis of biopsy specimens, tissues, and CSF to visualize the amoeba, along with computerized tomography (CT) or magnetic resonance imaging (MRI) to identify abnormalities, swelling, or bleeding of the brain [23,32-36]; however, since most patients exhibit symptoms similar to viral or bacterial meningitis, physicians may not consider an amoebic pathogen in the differential diagnosis. Furthermore, local testing laboratories may not be equipped with proper testing technology for accurate diagnosis [37]. Delays between pathogen exposure and therapeutic intervention are so crucial because they may contribute to the high mortality rate. In fact, authors reporting a successful patient outcome after hypothermia primarily attributed survival to rapid diagnosis made within one hour of receiving the CSF specimen, despite nonspecific symptoms, and recommended that medical pathologists consider the possibility of $N$. fowleri in suspected meningitis cases during the summer months [8]. Additionally, index of suspicion for PAM should be higher for physicians in certain regions: A quarter of all PAM cases in the United States occur in Florida likely due to environmental and climate factors, with Orange County having an especially high burden [38]. However, PAM has also been reported as far north as Minnesota [38], so physicians should not rule out the possibility of PAM diagnosis solely based on geography. Rather, it is the hope and intent of the authors that this publication would serve to heighten all physicians' awareness and index of suspicion for this rare illness, with the ultimate goal of providing vital treatment to those who need it.

\section{Limitations}

A major limitation of this study, and all studies investigating $N$. fowleri, is the lack of data on the incidences and management of such patients; the small number of studies reporting adjunctive hypothermia therapy especially limits the conclusions that can be drawn as well as the generalizability of these results. Furthermore, details pertaining to treatment regimens were not consistently reported across studies, including two studies that did not state the temperature of therapeutic hypothermia $[10,11]$, which prevents scientific rigor in identifying successful treatment parameters. Future studies and case reports should document the full details of hypothermic conditions, including the timing and duration of administration to fully characterize its benefits. Lastly, further in vitro and in vivo analyses are needed to study the impact of pharmaceutical agents and medical management techniques at each stage of the course of the disease.

\section{Conclusions}

N. fowleri infection is rare and difficult to treat because of its nonspecific symptoms with sudden onset. Our review of the literature demonstrated that therapeutic hypothermia is a relatively new adjunctive treatment that may have comparable mortality rate for $N$. fowleri patients who receive traditional therapies alone. Further studies and careful documentation of hypothermic conditions in real-world conditions may shed light on the utility of therapeutic hypothermia as a concomitant therapy to improve survival and neurological outcomes.

\section{Funding}

The United Hospital Foundation supported the preparation of the manuscript.

\section{Acknowledgments}

The authors acknowledge Superior Medical Experts for research and drafting assistance.

\section{Supplemental Digital Content}

Supplemental digital content Table 1.

\section{Conflicts of Interest}

$\mathrm{PH}$ and $\mathrm{AM}$ contract with Superior Medical Experts. No conflicts of interest were declared for the remaining authors.

\section{References}

1. Yoder JS, Eddy BA, Visvesvara GS, et al. (2010) The epidemiology of primary amoebic meningoencephalitis in the USA, 1962-2008. Epidemiol Infect 138: 968-975.

2. https://www.cdc.gov/parasites/naegleria/general.html

3. Grace E, Asbill S, Virga K (2015) Naegleria fowleri: Pathogenesis, diagnosis, and treatment options. Antimicrobial Agents and Chemotherapy 59: 6677-6681.

4. Jarolim KL, McCosh JK, Howard MJ, et al. (2000) A light microscopy study of the migration of Naegleria fowleri from the nasal submucosa to the central nervous system during the early stage of primary amebic meningoencephalitis in mice. J Parasitol 86: 50-55.

5. Marciano-Cabral F, Cabral GA (2007) The immune response to Naegleria fowleri amebae and pathogenesis of infection. FEMS Immunol Med Microbiol 51: 243-259.

6. John DT, Cole TB Jr, Bruner RA (1985) Amebostomes of Naegleria fowleri. J Protozool 32: 12-19.

7. Linam WM, Ahmed M, Cope JR, et al. (2015) Successful treatment of an adolescent with Naegleria fowleri primary amebic meningoencephalitis. Pediatrics 135: e744-e748. 
Citation: Defillo A, Nussbaum PE, Hariharan P, et al. (2021) Hypothermia as an Adjunctive Treatment in Pediatric Patients with Naegleria fowleri: A Systematic Review. J Pediatr Neurol Neurosci 5(1):105-110

8. Dunn AL, Reed T, Stewart C, et al. (2016) Naegleria fowleri That Induces Primary Amoebic Meningoencephalitis: Rapid Diagnosis and Rare Case of Survival in a 12-Year-Old Caucasian Girl. Lab Med 47: 149-154.

9. Heggie TW, Kupper T (2017) Surviving Naegleria fowleri infections: A successful case report and novel therapeutic approach. Travel Med Infect Dis 16: 49-51.

10. Cope JR, Conrad DA, Cohen N, et al. (2016) Use of the Novel Therapeutic Agent Miltefosine for the Treatment of Primary Amebic Meningoencephalitis: Report of 1 Fatal and 1 Surviving Case. Clinical infectious diseases: An official publication of the Infectious Diseases Society of America 62: 774-776.

11. Stowe RC, Pehlivan D, Friederich KE, et al. (2017) Primary Amebic Meningoencephalitis in Children: A Report of Two Fatal Cases and Review of the Literature. Pediatric Neurology 70: 75-79.

12. Stevens AR, Shulman ST, Lansen TA, et al. (1981) Primary amoebic meningoencephalitis: $A$ report of two cases and antibiotic and immunologic studies. J Infect Dis 143: 193-199.

13. Kidney DD, Kim SH (1998) CNS infections with free-living amebas: Neuroimaging findings. AJR Am J Roentgenol 171: 809-812.

14. Movahedi Z, Shokrollahi MR, Aghaali M, et al. (2012) Primary amoebic meningoencephalitis in an Iranian infant. Case Rep Med 2012: 782854.

15. Kemble SK, Lynfield R, DeVries AS, et al. (2012) Fatal Naegleria fowleri infection acquired in Minnesota: possible expanded range of a deadly thermophilic organism. Clin Infect Dis 54: 805809.

16. Roy SL, Metzger R, Chen JG, et al. (2014) Risk for transmission of Naegleria fowleri from solid organ transplantation. Am J Transplant 14: 163-171.

17. Mittal N, Mahajan L, Hussain Z, et al. (2019) Primary amoebic meningoencephalitis in an infant. Indian J Med Microbiol 37: 120-122.

18. Sood A, Chauhan S, Chandel L, et al. (2014) Prompt diagnosis and extraordinary survival from Naegleria fowleri meningitis: A rare case report. Indian J Med Microbiol 32: 193-196.

19. Seidel JS, Harmatz P, Visvesvara GS, et al. (1982) Successful treatment of primary amebic meningoencephalitis. N Engl J Med 306: 346-348.

20. Singh SN, Patwari AK, Dutta R, et al. (1998) Naegleria meningitis. Indian Pediatr 35: 1012-1015.

21. Rai R, Singh DK, Srivastava AK, et al. (2008) Primary amebic meningoencephalitis. Indian Pediatr 45: 1004-1005.

22. Vargas-Zepeda J, Gómez-Alcalá AV, Vásquez-Morales JA, et al. (2005) Successful treatment of Naegleria fowleri meningoencephalitis by using intravenous amphotericin $B$, fluconazole and rifampicin. Arch Med Res 36: 83-86.
23. Visvesvara GS, Moura H, Schuster FL (2007) Pathogenic and opportunistic free-living amoebae: Acanthamoeba spp., Balamuthia mandrillaris, Naegleria fowleri, and Sappinia diploidea. FEMS Immunol Med Microbiol 50: 1-26.

24. (1992) Prevention CfDCa: From the Centers for Disease Control. Primary amebic meningoencephalitis-North Carolina, 1991. JAMA 268: 862-863.

25. (2003) Prevention CfDCa: Primary amebic meningoencephalitis-Georgia, 2002. MMWR Morb Mortal Wkly Rep 52: 962-964.

26. Kramer MH, Lerner CJ, Visvesvara GS (1997) Kidney and liver transplants from a donor infected with Naegleria fowleri. J Clin Microbiol 35: 1032-1033.

27. Cope JR, Ratard RC, Hill VR, et al. (2015) The first association of a primary amebic meningoencephalitis death with culturable Naegleria fowleri in tap water from a US treated public drinking water system. Clin Infect Dis 60: e36-e42.

28. Yenari MA, Han HS (2012) Neuroprotective mechanisms of hypothermia in brain ischaemia. Nat Rev Neurosci 13: 267-278.

29. Zaongo SD, Shaio MF, Ji DD (2018) Effects of Culture Media on Naegleria fowleri Growth at Different Temperatures. J Parasitol 104: 451-456.

30. Goudot S, Herbelin P, Mathieu L, et al. (2012) Growth dynamic of Naegleria fowleri in a microbial freshwater biofilm. Water research 46: 3958-3966.

31. Diaz J (2012) Seasonal primary amebic meningoencephalitis (PAM) in the south: Summertime is PAM time. J La State Med Soc 164: 148-155.

32. (2008) Prevention CfDCa: Primary amebic meningoencephalitis-Arizona, Florida, and Texas, 2007. MMWR Morb Mortal Wkly Rep 57: 573-577.

33. https://www.cdc.gov/parasites/naegleria/diagnosis.html

34. Hebbar S, Bairy I, Bhaskaranand N, et al. (2005) Fatal case of naegleria fowleri meningo-encephalitis in an infant: Case report. Ann Trop Paediatr 25: 223-226.

35. Martínez A (1985) Free-living amebas: Natural history, prevention, diagnosis, pathology, and treatment of Disease. Boca Raton, FL: CRC Press; USA.

36. https://www.mayoclinic.org/diseases-conditions/naegleria-infection/diagnosis-treatment/drc-20375474

37. Pugh JJ, Levy RA (2016) Naegleria fowleri: Diagnosis, pathophysiology of brain inflammation, and antimicrobial treatments. ACS chemical neuroscience 7: 1178-1179.

38. Budge PJ, Lazensky B, Van Zile KW, et al. (2013) Primary amebic meningoencephalitis in Florida: A case report and epidemiological review of Florida cases. J Environ Health 75: 26-31.

DOI: $10.36959 / 595 / 417$

Copyright: (c) 2021 Defillo A, et al. This is an open-access article distributed under the terms of the Creative Commons Attribution License, which permits unrestricted use, distribution, and reproduction in any medium, provided the original author and source are credited. 